\title{
Erratum: Three-Dimensional Dynamics of a Magnetic Hopfion Driven by Spin Transfer Torque [Phys. Rev. Lett. 124, 127204 (2020)]
}

Yizhou Liu, Wentao Hou, Xiufeng Han, and Jiadong Zang

(a) (Received 20 September 2020; published 5 October 2020)

DOI: 10.1103/PhysRevLett.125.159901

In the Letter, a factor of 2 is missing in the expressions of $S_{0}^{x}$ and $S_{0}^{y}$ in Eq. (1). The correct form of Eq. (1) reads

$$
\begin{aligned}
& S_{0}^{x}=\frac{x}{r} \sin 2 f+\frac{2 y z}{r^{2}} \sin ^{2} f, \\
& S_{0}^{y}=\frac{y}{r} \sin 2 f-\frac{2 x z}{r^{2}} \sin ^{2} f, \\
& S_{0}^{z}=\cos 2 f+\frac{2 z^{2}}{r^{2}} \sin ^{2} f .
\end{aligned}
$$

Note that the analytical analysis and numerical results presented were performed based on the correct expression, and the conclusion of the Letter is not affected. 